Discussiones Mathematicae

Note

\title{
STAR-CYCLE FACTORS OF GRAPHS
}

\author{
Yoshimi EgawA \\ Tokyo University of Science, Shinjuku-Ku, Tokyo, Japan \\ MIKIO KANO ${ }^{1}$ \\ Ibaraki University, Hitachi, Ibaraki, Japan \\ e-mail: kano@max.ibaraki.ac.jp \\ AND \\ ZHENG YAN \\ Ibaraki University, Hitachi, Ibaraki, Japan \\ e-mail: yanzhenghubei@163.com
}

\begin{abstract}
A spanning subgraph $F$ of a graph $G$ is called a star-cycle factor of $G$ if each component of $F$ is a star or cycle. Let $G$ be a graph and $f: V(G) \rightarrow$ $\{1,2,3, \ldots\}$ be a function. Let $W=\{v \in V(G): f(v)=1\}$. Under this notation, it was proved by Berge and Las Vergnas that $G$ has a star-cycle factor $F$ with the property that (i) if a component $D$ of $F$ is a star with center $v$, then $\operatorname{deg}_{F}(v) \leq f(v)$, and (ii) if a component $D$ of $F$ is a cycle, then $V(D) \subseteq W$ if and only if $i s o(G-S) \leq \sum_{x \in S} f(x)$ for all $S \subset V(G)$, where $i s o(G-S)$ denotes the number of isolated vertices of $G-S$. They proved this result by using circulation theory of flows and fractional factors of graphs. In this paper, we give an elementary and short proof of this theorem.
\end{abstract}

Keywords: star factor, cycle factor, star-cycle factor, factor of graph.

2010 Mathematics Subject Classification: 05C70.

\section{REFERENCES}

\footnotetext{
${ }^{1}$ The author was supported by Japan Society for the Promotion of Science, Grant-in-Aid for Scientific Research (C).
} 
[1] J. Akiyama and M. Kano, Factors and Factorizations of Graphs (Lecture Notes in Math. 2031, Springer, 2011).

[2] A. Amahashi and M. Kano, On factors with given components, Discrete Math. 42 (1983) $1-6$.

doi:10.1016/0012-365X(82)90048-6

[3] C. Berge and M. Las Vergnas, On the existence of subgraphs with degree constraints, Nederl. Akad. Wetensch. Indag. Math. 40 (1978) 165-176.

doi:10.1016/1385-7258(78)90034-3

[4] M. Las Vergnas, An extension of Tutte's 1-factor theorem, Discrete Math. 23 (1978) 241-255.

doi:10.1016/0012-365X(78)90006-7

[5] W.T. Tutte, The 1-factors of oriented graphs, Proc. Amer. Math. Soc. 4 (1953) 922-931.

doi: $10.2307 / 2031831$

Received 28 August 2012

Revised 13 December 2012

Accepted 28 December 2012 